\title{
The importance of dust in cooling and heating the InterGalactic Medium
}

\author{
L. A. Montier ${ }^{1}$ and M. Giard ${ }^{1}$ \\ Centre d’Études Spatiales des Rayonnements, 9 avenue du Colonel Roche, 31022 Toulouse, France \\ Received 19 September 2003 / Accepted 18 December 2003
}

\begin{abstract}
Recent observations at low and high redshift seem to confirm the presence of dust in the InterGalactic Medium (IGM) and especially in the IntraCluster Medium (ICM), at very low abundances. We have studied the impact of this dust on the IGM conditions, in terms of heating and cooling. Firstly we have described the dust emission by an analytical model, and we have proved that the dust IR emission can be considered as the dominant cooling agent of the ICM, when the temperature is greater than $T=10^{7} \mathrm{~K}$ and the dust-to-gas mass ratio exceeds a value of $Z_{\mathrm{d}}=2 \times 10^{-5}$. This cooling process is more efficient for small grains, and fills the gap between the cooling by free-free emission at $T>10^{8} \mathrm{~K}$ and other cooling processes such as recombination, collisional ionisation and collisional excitation at $T<10^{5} \mathrm{~K}$. Secondly, when the UV Background is strong enough, $J_{\mathrm{UV}}(v) \geq 10^{-22}(\mathrm{v} /(13.6 \mathrm{eV}))^{-1} \mathrm{erg} \mathrm{s}^{-1} \mathrm{~cm}^{-2} \mathrm{sr}^{-1} \mathrm{~Hz}^{-1}$, and the temperature is low enough, $T_{\mathrm{e}} \leq 10^{5} \mathrm{~K}$, dust grains become an efficient heating agent of the IGM. Moreover these two opposite processes due to dust have opposite impact on the IGM at different scales: the cooling should be dominant at large scale (in the ICM), whereas the heating is efficient at small scale (near quasars or star formation regions). The issue of the dust replenishment of the IGM is also considered, taking into account the process of galactic dust ejection and intergalactic dust production. The recent observations of an intergalactic star population and the estimate of the reionisation (pop III stars) at $z=12-20$, both point toward dust pollution of the IGM early in the cosmic history, with possible important consequences for the evolution scenarii of the Universe.
\end{abstract}

Key words. galaxies: intergalactic medium - ISM: dust extinction - galaxies: cluster: general

\section{Introduction}

The presence of dust in the intergalactic medium (IGM) was first pointed out by Zwicky et al. (1957) and Karachentsev \& Lipovetskii (1969), when observing the extinction of distant clusters behind rich galaxy clusters. Similar observations have been made by Bogart \& Wagoner (1973), and Boyle et al. (1988) when counting quasars behind rich clusters. Recently dust has been detected in emission toward clusters of galaxies in the IRAS data (Wise 1993), and toward the Coma cluster (Stickel et al. 1998, 2002). A dust extinction curve at high redshift has been measured by Motta et al. (2002), using the differential image of a quasar along different paths through the IGM by a galaxy lens, attesting the presence of dust early in the cosmic history. Naturally all these claims remain controversial (see Maoz 1995; Pointecouteau 1999) and have to be confirmed by further studies. Nevertheless, the recent observations by the Wilkinson Microwave Anisotropy Probe (WMAP) (Bennet et al. 2003a) seem to indicate an early epoch of reionisation of $z=17 \pm 5$ (Spergel et al. 2003), opening a new area of massive dust pollution by stars of population III, as studied by Nozawa et al. (2003).

However even at a very low abundance, dust remains one of the important actors of the IGM evolution. It could have

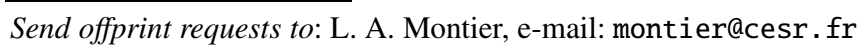

important implications on cosmology, by affecting the count of galaxies and quasars at high redshift (see Aguirre 1999) because of dust extinction, or by affecting the measurement of the cosmic microwave background (CMB) at small scales, and the cosmic infrared backgrounds (CIB) because of the selfemission by the dust. Moreover the estimate of the SZ effect toward clusters of galaxies could be affected too, considering the high sensitivity of future missions like Planck.

Dust may also have a direct impact on the properties of the IGM. First revealed by Dwek \& Werner (1981, hereafter DW81) and Dwek et al. (1987) around supernovae, the dust emission represents an important cooling agent in such a hot medium, and might have a similar impact on the intracluster medium.

In this work we will first present in Sect. 2 a simple modeling of the intracluster medium to point out the potential importance of dust cooling compared to free-free cooling. In Sect. 3, we will then detail the impact of dust on this type of medium by taking into account the dust heating and the usual atomic processes of cooling to exhibit some spatial and temperature range of dust cooling efficiency. We will finally discuss in Sects. 4 and 5 the problem of the dust abundance in the IGM, and the potential impact of dust on the evolution of the structures at different scales of the universe. 


\section{Dust emission from the intracluster medium}

In this section, we will focus on the intracluster medium (hereafter ICM), which makes the denser fraction of the IGM. The ICM represents the nodes of the global network of the matter distribution in the Universe (e.g. as observed by the $2 \mathrm{dF}$ Galaxy Redshift Survey, Percival et al. 2001). Because of the nature of these nodes, described as infalling and virialized structures, the ICM has typical properties of high density $\left(n_{\mathrm{e}} \approx 10^{-3} \mathrm{~cm}^{-3}\right)$ and temperature $\left(10^{6} \leq T_{\mathrm{e}} \leq 10^{8} \mathrm{~K}\right)$, compared with the rest of the IGM $\left(n_{\mathrm{e}} \approx 10^{-6} \mathrm{~cm}^{-3}\right.$ and $\left.10^{4} \leq T_{\mathrm{e}} \leq 10^{6} \mathrm{~K}\right)$. We will quantitatively investigate in which conditions the dust can become an important cooling agent of the ICM.

\subsection{Energetic balance}

One can first note that the dust is never at equilibrium in the ICM. The smallest grains are stochasticaly heated, and their temperature has to be modeled with a dust temperature distribution as describe in Dwek (1986). But in our case, we neglect this effect for the reason of simplicity, and assume thermal equilibrium for all the dust components. The detailed energetic balance of the grain is then given by:

$H_{\text {coll }}\left(a, T_{\mathrm{e}}, n_{\mathrm{e}}\right)=\Lambda\left(a, T_{\mathrm{d}}\right)$

where $H_{\text {coll }}$ is the collisional heating of the grain, and $\Lambda$ is the cooling rate due to the dust thermal emission, as detailed below. We neglect here the radiative heating due to the UV and Cosmic Microwave Background, given the low radiation field in the intracluster medium, as described in Sect. 3.2.

The dust cooling will occur via thermal infrared emission.

$\Lambda\left(a, T_{\mathrm{d}}\right)=4 \pi^{2} a^{2} \int_{v} Q_{\mathrm{abs}}(v, a) B_{v}\left(T_{\mathrm{d}}\right) \mathrm{d} v$

where $a$ is the grain radius, $v$ is the frequency, $Q_{\mathrm{abs}}$ is the absorption coefficient given by Draine \& Lee (1984) for silicate and graphite grains, $B_{v}$ is the Planck function, and $T_{\mathrm{d}}$ is the equilibrium dust temperature.

The collisions with electrons will dominate over those with protons because for the same energy, the electrons have much higher velocities, implying a higher collision rate. We will use the collisional heating rate obtained by DW81 which takes into account the fact that electrons which impinge on a dust grain will penetrate deeper if they have more energy, and reversely they will deposit only a fraction of their energy if the grain is too small. Consequently, the grain heating derived by DW81 depends on the grain radius:

$H_{\text {coll }}\left(a, T_{\mathrm{e}}, n_{\mathrm{e}}\right)= \begin{cases}5.38 \times 10^{-18} n_{\mathrm{e}} a^{2} T_{\mathrm{e}}^{3 / 2} & x^{*}>4.5 \\ 3.37 \times 10^{-13} n_{\mathrm{e}} a^{2.41} T_{\mathrm{e}}^{0.88} & x^{*}>1.5 \\ 6.48 \times 10^{-6} n_{\mathrm{e}} a^{3} & x^{*} \leq 1.5\end{cases}$

where $H_{\text {coll }}$ is in $\mathrm{erg} \mathrm{s}^{-1}, a(\mu \mathrm{m})$ is the grain radius, $n_{\mathrm{e}}\left(\mathrm{cm}^{-3}\right)$ the electronic density, $T_{\mathrm{e}}$ the electronic temperature, and $x^{*}=$ $2.71 \times 10^{8} a^{2 / 3} / T_{\mathrm{e}}$. The electronic collisional heating is less efficient below $T_{\mathrm{e}}=10^{8} \mathrm{~K}$ for a grain size of $a=0.5 \mu \mathrm{m}$ as shown in the upper curve of Fig. 1, and this limit decreases when the grain size becomes smaller.

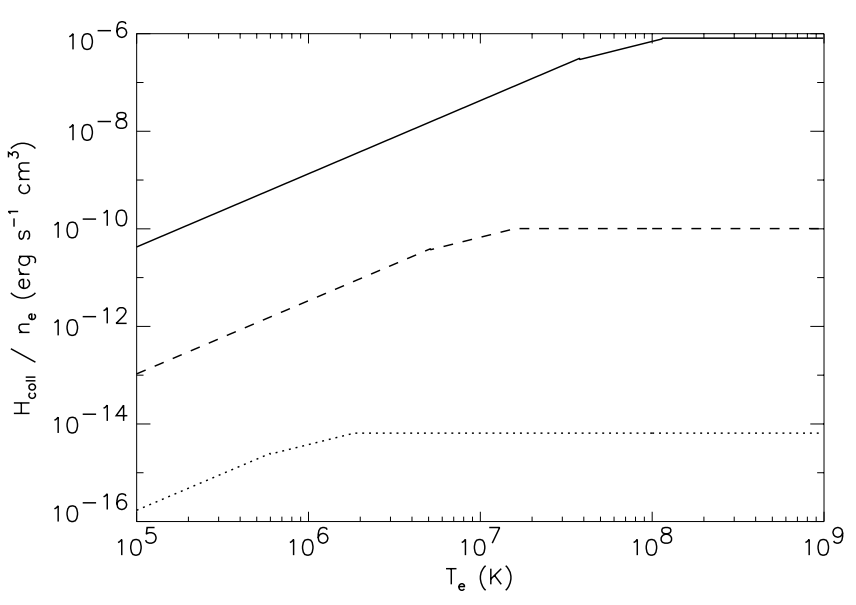

Fig. 1. Heating function of the grain due to electronic collisions, for various grain sizes: $a=0.5 \mu \mathrm{m}$ (solid line), $a=0.025 \mu \mathrm{m}$ (dashed line), $a=0.001 \mu \mathrm{m}$ (dotted line).

We can then deduce the dust equilibrium temperature $T_{\mathrm{d}}$ by solving Eq. (1), but we have to keep in mind the limitation of such an assumption of equilibrium in the context of small grains stochasticaly heated by electrons. In this case, the dusttemperature distribution peaks below the equilibrium value, but extends to extremely higher temperatures $\left(T_{\mathrm{d}} \approx 1000 \mathrm{~K}\right)$. The consequence of such a temperature distribution is the broadening of the IR-spectrum. This will have an impact on the detection of the dust emission, but it does not change the integrated flux over the spectrum (DW81).

If we use a simple power law with index $\beta$ for the dust emissivity then the energetic balance of a single grain reads:

$H_{\text {coll }}=T_{\mathrm{d}}^{\beta+4} \quad \frac{8 \pi^{2} a^{3} k^{\beta+4}}{c^{2} h^{\beta+3} v_{0}^{\beta}} \frac{Q_{\mathrm{abs}}\left(v_{0}, a\right)}{a} \int_{0}^{\infty} \frac{x^{\beta+3}}{\mathrm{e}^{x}-1} \mathrm{~d} x$

where we have written $Q_{\mathrm{abs}}(v, a)=Q_{\mathrm{abs}}\left(v_{0}, a\right)\left(\frac{v}{v_{0}}\right)^{\beta}, Q_{\mathrm{abs}}$ is the absorption coefficient given by Draine \& Lee (1984) for silicate and graphite grains, $v_{0}$ is a reference frequency, $T_{\mathrm{d}}$ is the equilibrium dust temperature, $h$ is Planck's constant, $c$ is the velocity of light, $k$ is Boltzmann's constant, and $x$ stands for $h v / k T$.

The solution can then be expressed as follows:

$T_{\mathrm{d}}=C\left(a, T_{\mathrm{e}}, \beta\right)\left(\frac{n_{\mathrm{e}}}{2 \times 10^{-3} \mathrm{~cm}^{-3}}\right)^{1 /(\beta+4)} \mathrm{K}$

where $C\left(a, T_{\mathrm{e}}, \beta\right)$ is a coefficient depending on the grain size $a$, the electronic temperature $T_{\mathrm{e}}$ and the index $\beta$, and $n_{\mathrm{e}}$ is the electronic density. Above $T_{\mathrm{e}} \approx 2 \times 10^{7} \mathrm{~K}$, the resulting dust equilibrium temperature becomes independent of the electronic temperature and the grain size for almost the full size range of our dust model.

As far as $\beta$ is concerned, the far infrared and submillimeter investigations of the Pronaos balloon in various galactic regions (Dupac et al. 2003) have demonstrated that the dust spectral index spans a range from 1 to 2.5 . Moreover, the same authors show that the index is anticorrelated with the dust temperature, the higher temperatures $(T \approx 60 \mathrm{~K})$ corresponding to low indices $(\beta=1)$. At centimeter wavelengths the dust emission extracted from the WMAP one year data shows $\beta=2.2$ 
(Bennett et al. 2003b). Here the exact value of $\beta$ is not critical since the dust cooling will scale as $L_{\mathrm{IR}} \propto T^{4+\beta}$. We will use $\beta=2$. With this hypothesis the grain temperature for $T_{\mathrm{e}} \geq 2 \times 10^{7} \mathrm{~K}$ reads (see also Dwek et al. 1990):

$T_{\mathrm{d}} \approx 20\left(\frac{n_{\mathrm{e}}}{2 \times 10^{-3} \mathrm{~cm}^{-3}}\right)^{1 / 6} \quad \mathrm{~K}$.

\subsection{Dust emission from a massive cluster}

The IntraCluster Medium in massive clusters seems to be the best candidate to observe the dust emission, because of its high density and its high electronic temperature. This is why we have calibrated our simulation on the Coma cluster A1656. We use an isothermal spherical model, with a high electronic temperature $T_{\mathrm{e}}$, and an electronic density profil $n_{\mathrm{e}}(R)$ given by a King Model

$n_{\mathrm{e}}(R)=n_{\mathrm{e} 0}\left(1+\left(\frac{R}{R_{g}}\right)^{2}\right)^{-\frac{3}{2} \alpha}$,

where $n_{\mathrm{e} 0}$ is the central density, $R_{g}$ the core radius, and $\alpha$ the power coefficient of King's law. The dust density profile $n_{\mathrm{d}}(R)$ is chosen proportional to the electronic one, without local fluctuations, and given by:

$n_{\mathrm{d}}(R)=Z_{\mathrm{d}}^{\mathrm{ICM}} \frac{\mu_{\mathrm{H}} m_{\mathrm{H}}}{<m_{\mathrm{d}}>}\left(\frac{n_{\mathrm{H}}}{n_{\mathrm{e}}}\right) n_{\mathrm{e}}(R)$

where $n_{\mathrm{H}} / n_{\mathrm{e}}=0.86$ is the ratio of the hydrogen to electronic density of the gas that consists of totally ionized hydrogen and helium in agreement with the cosmic abundance $(X=0.75$, $Y=0.24) ; \mu_{\mathrm{H}}=1.32$ is the mean atomic weight per $\mathrm{H}$ atom; $m_{\mathrm{H}}$ is the mass of the hydrogen atom; $\left\langle m_{\mathrm{d}}\right\rangle$ is the mean dust weight of a graphite-silicate grain mixture integrated over the whole size range $0.001<a<0.5 \mu \mathrm{m}$, with a grain size distribution given by $N(a) \mathrm{d} a=a^{-3.5} \mathrm{~d} a$ (Mathis et al. 1977, hereafter $\mathrm{MRN}$ ); and $Z_{\mathrm{d}}^{\mathrm{ICM}}=M_{\mathrm{d}} / M_{\mathrm{g}}$ is the dust-to-gas mass ratio of the ICM.

The expression of the IR luminosity integrated over the whole galaxy cluster is given by:

$L_{\mathrm{IR}}^{\mathrm{ICM}}=16 \pi^{3} \iiint n_{\mathrm{d}} a^{2} Q_{\mathrm{abs}} B_{v}\left(T_{\mathrm{d}}\right) R^{2} N(a) \mathrm{d} a \mathrm{~d} v \mathrm{~d} R$.

Using the energetic balance of a grain, we obtain:

$L_{\mathrm{IR}}^{\mathrm{ICM}}=4 \pi \iint n_{\mathrm{d}}(R) H_{\text {coll }}\left(a, T_{\mathrm{e}}, n_{\mathrm{e}}\right) R^{2} N(a) \mathrm{d} a \mathrm{~d} R$.

The thermal conditions of this galaxy cluster $T_{\mathrm{e}}=10^{8} \mathrm{~K}$ and the grain size range of our dust model lead to a simplification of the collisional heating function, corresponding to the third case in the Eq. (3). We obtain an analytical expression of the IR luminosity (in erg s${ }^{-1}$ ) integrated over the whole cluster and all grain sizes, which depends only on the electronic density, the dust-to-gas mass ratio and a geometrical coefficient:

$L_{\mathrm{IR}}^{\mathrm{ICM}}=3.7 \times 10^{45}\left(\frac{Z_{\mathrm{d}}^{\mathrm{ICM}}}{10^{-5}}\right)\left(\frac{n_{\mathrm{e} 0}}{10^{-3} \mathrm{~cm}^{-3}}\right)^{2} G_{\mathrm{eom}}$ where

$G_{\mathrm{eom}}=\left(\frac{R_{g}}{\mathrm{Mpc}}\right)^{3} f\left(\frac{R_{\mathrm{c}}}{R_{g}}, \alpha\right)$

with $R_{\mathrm{c}}$ the critical radius, i.e. the maximum radius of the spatial integration given by $n_{\mathrm{e}}\left(R_{\mathrm{c}}\right)=10^{-3} n_{\mathrm{e}}\left(R_{g}\right)$, that means approximately $R_{\mathrm{c}} \approx 9.95 R_{g}$ (Voshchinnikov \& Khersonskij 1984). The term $f$ is the following function:

$f(\delta, \alpha)=\int_{0}^{\delta}\left[1+\zeta^{2}\right]^{-3 \alpha} \zeta^{2} \mathrm{~d} \zeta$

\subsection{Comparing IR and $X$-ray luminosities}

To estimate the efficiency of the dust IR luminosity in the cooling process, it has to be compared to the X-ray luminosity (Bremsstrahlung), considered as the main cooling agent of the ICM. The expression of the X-ray luminosity integrated over the cluster is given (in $\operatorname{erg~s}^{-1}$ ) by Mushotzky et al. (1978)

$L_{\mathrm{X}}^{\mathrm{ICM}}=6.6 \times 10^{45} \sqrt{\frac{T_{\mathrm{e}}}{10^{8} \mathrm{~K}}}\left(\frac{n_{\mathrm{e} 0}}{10^{-3} \mathrm{~cm}^{-3}}\right)^{2} G_{\mathrm{eom}}$

where $T_{\mathrm{e}}$ is the electronic temperature, and $G_{\text {eom }}$ has the same expression as for the IR luminosity. The ratio of Eqs. (11) and (13) gives a good estimation of the relative contributions by IR to X-ray luminosities which depends only on the electronic temperature and the dust-to-gas mass ratio.

$\frac{L_{\mathrm{IR}}^{\mathrm{ICM}}}{L_{\mathrm{X}}^{\mathrm{ICM}}}=0.56\left(\frac{Z_{\mathrm{d}}^{\mathrm{ICM}}}{10^{-5}}\right)\left(\frac{T_{\mathrm{e}}}{10^{8} \mathrm{~K}}\right)^{-1 / 2}$

This result, which is valid only for high electronic temperatures $T_{\mathrm{e}}>10^{7} \mathrm{~K}$ (rich clusters), shows that the IR luminosity of intracluster dust can be higher than the X-ray emissivity of the gas for a dust-to-gas mass ratio as low as $10^{-4}-10^{-5}$. This is $10^{-2}-10^{-3}$ lower than the typical dust-to-gas mass ratio in our Milky Way. In order to establish the cooling efficiency of the dust emission in the intergalactic medium for temperatures lower than $T_{\mathrm{e}}=10^{7} \mathrm{~K}$, it is important to use the full expression of the dust heating in Eq. (3), and to compare the integrated dust cooling function with oher cooling and heating mechanisms. This is done in Sect. 3.

\section{Efficiency of the dust cooling in the IGM}

We have already shown the efficiency of the dust cooling compared to the free-free cooling in a simple case. We will now examine in detail the impact of the dust, in regard to both aspects of cooling and heating: first we will compare the cooling by dust emission with the heating by the photoelectric effect on dust in order to identify domains of dust cooling or heating, depending on the temperature, the density and the UV background of the IGM; then we will compare the cooling by dust emission with atomic processes of cooling, that are commonly known as efficient cooling agents of the IGM. 


\subsection{Dust heating and cooling}

When dust grains are placed in Lyman- $\alpha$ absorption systems, with strong UV Background (hereafter UVB), they may heat the medium by the photoelectric effect. The photoelectric effect consists of the emission of an electron to the gas after absorption of a very energetic single photon (Weingartner \& Draine 2001). This heating process by dust is not negligible and can affect the history of the IGM, as first claimed by Nath et al. (1999). Inoue \& Kamaya (2003) showed that at very high redshift from $z=1.5$ to $z=6$ the photoelectric heating by grains can dominate other heating mechanisms, like photoionisation, by a factor 3 to 10 , depending on the grain size; the smaller the grain, the better the heating.

An estimation of the photoelectric dust heating rate was made following the formalism developped by Weingartner \& Draine (2001) and applied to a grain mixture of graphite and silicate as defined by the MRN model. The grain charge was calculated assuming equilibrium between the electron collision rate $R_{\mathrm{e}}$ (depending on the electronic density, the grain size and the temperature) and the photoelectric ejection rate $R_{\mathrm{pe}}$ (depending on the grain size and the UVB). We have used Bake \& Tielens' (1994) values for the electron escape length $l_{\mathrm{e}}=10^{-7} \mathrm{~cm}$ and the photon attenuation length $l_{\mathrm{a}}=10^{-6} \mathrm{~cm}$ into the grain. The UV background spectrum is modeled by a power-law given by Kitayama et al. (2001)

$J_{v}=J_{21}\left(\frac{v}{v_{0}}\right)^{-1}$

where $v_{0}$ is the frequency of ionization of hydrogen $\left(h v_{0}=\right.$ $13.6 \mathrm{eV}), J_{21}$ is the UV intensity at the Lyman limit of hydrogen in units of $10^{-21} \mathrm{erg} \mathrm{s}^{-1} \mathrm{~cm}^{-2} \mathrm{sr}^{-1} \mathrm{~Hz}^{-1}$. The coefficient $J_{21}$ has been estimated from recent reionisation models for redshifts $z=0$ to $z=20$, and has been determined to be around unity or less, depending on the redshift (Kitayama et al. 2001). Bajtlik et al. (1988), Bechtold (1994), or Giallongo et al. (1996) have estimated this coefficient at different redshifts near QSO's and found $J_{21}=10^{ \pm 0.5}$, but its value for the IGM is still highly uncertain. Its estimate near star formation regions can also be given by the parameter $G$ which is defined as the ratio of the total energy density in the radiation field and Habing's (1968) estimate of the average starlight energy density in the range $6-13.6(\mathrm{eV})$, the latter one being equal to $5.33 \times 10^{-14} \mathrm{erg} \mathrm{cm}^{-3}$. The relation between this two parameters in terms of total energy is given by $G=2.12 \times 10^{-2} J_{21}$. The photoelectric dust heating rate was finally estimated using the approximation of Inoue \& Kayama (2003), which is consistent with the Weingartner and Draine method.

We have compared this heating rate with the dust cooling rate derived in Sect. 2. This is shown in Fig. 2, where we draw contours for the difference between the two rates (in $\mathrm{erg} \mathrm{s}^{-1} \mathrm{~cm}^{-3}$ ), as a function of electronic temperature and UVB. This has been done for three grain sizes: $a=0.001$, 0.025 , and $0.5 \mu \mathrm{m}$, with a hydrogen density $n_{\mathrm{H}}=10^{-6} \mathrm{~cm}^{-3}$ (cf. Figs. 2-4, respectively). One can first remark that the steps in the contours are not due to numerical problems. They are the result of the quantification of the grain charge. The dust cooling is predominant in all cases for $T \geq 10^{5} \mathrm{~K}$ and $J_{21} \leq 10^{4}$. But

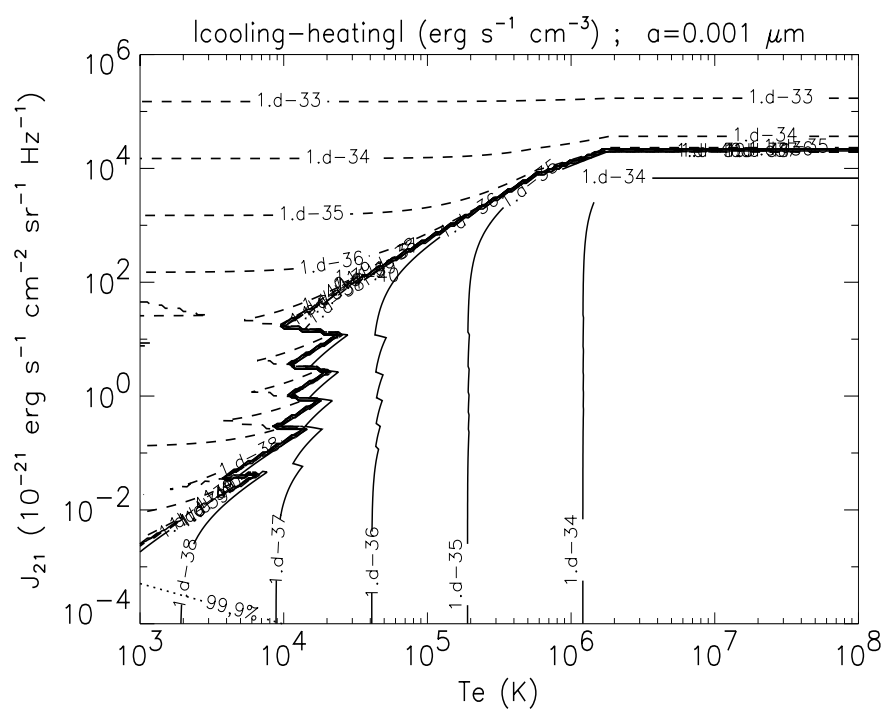

Fig. 2. Absolute value of the difference between the dust cooling rate and the photoelectric dust heating rate (in $\mathrm{erg} \mathrm{s}^{-1} \mathrm{~cm}^{-3}$ ) depending on the temperature and the UVB, for a grain size $a=0.001 \mu \mathrm{m}$ and a hydrogen density $n_{\mathrm{H}}=10^{-6} \mathrm{~cm}^{-3}$; cooling dominant (solid line) and heating dominant (dashed line). The level of $99.9 \%$ of ionisation is drawn as a dotted line.

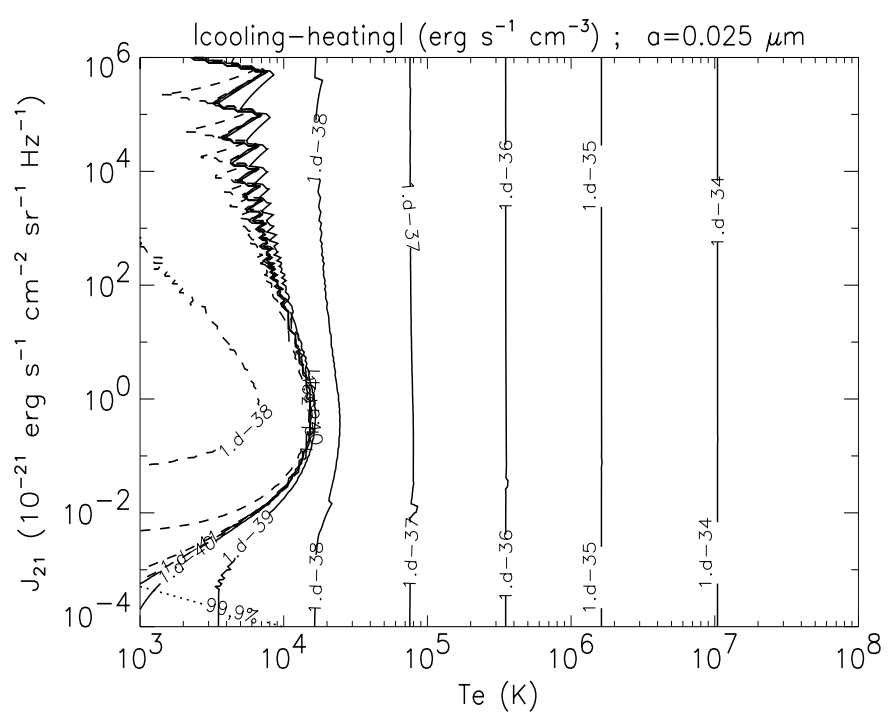

Fig. 3. The same as in Fig. 2 with a grain size $a=0.025 \mu \mathrm{m}$.

when the grain size increases, the efficiency of the photoelectric heating decreases rapidly, reducing the heating domain to the lower temperature and medium UV background. This domain corresponds to the IGM properties, near quasar or standard star formation regions. One can remark that the maximum value of the dust cooling rate remains the same at all grain sizes. But the higher is the grain radius, the higher is the temperature limit of the maximum level. This will be considered in detail in Sect. 3.2.

Another parameter is the density of the medium. On one hand the dust cooling is proportional to the dust density and to the electronic collision rate. On the other hand the photoelectric heating rate is naturally proportional to the dust density, and to the photoelectric yield. However the variation of the density, 


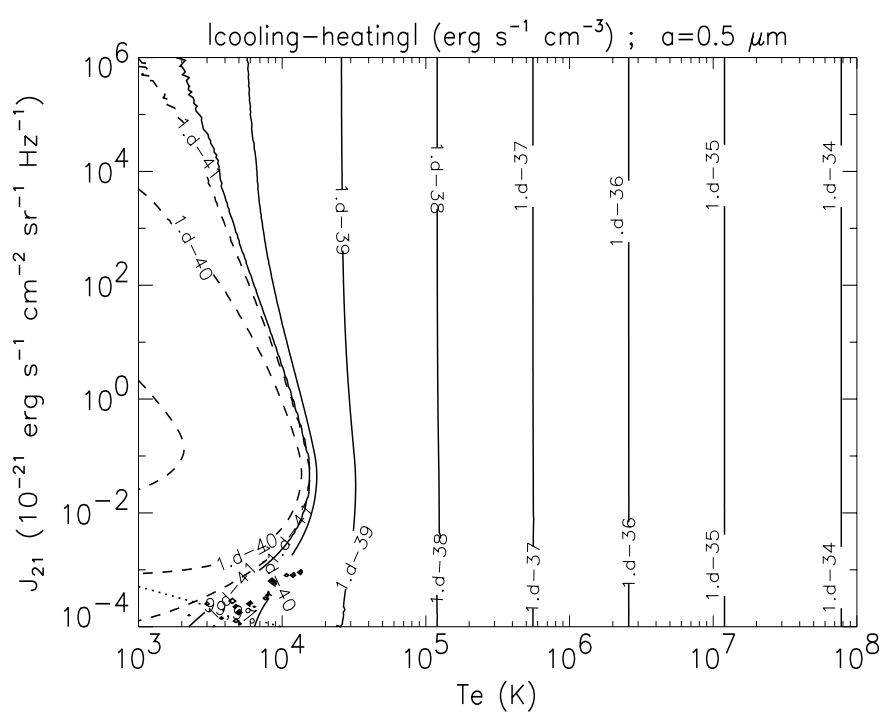

Fig. 4. The same as in Fig. 2 with a grain size $a=0.5 \mu \mathrm{m}$.

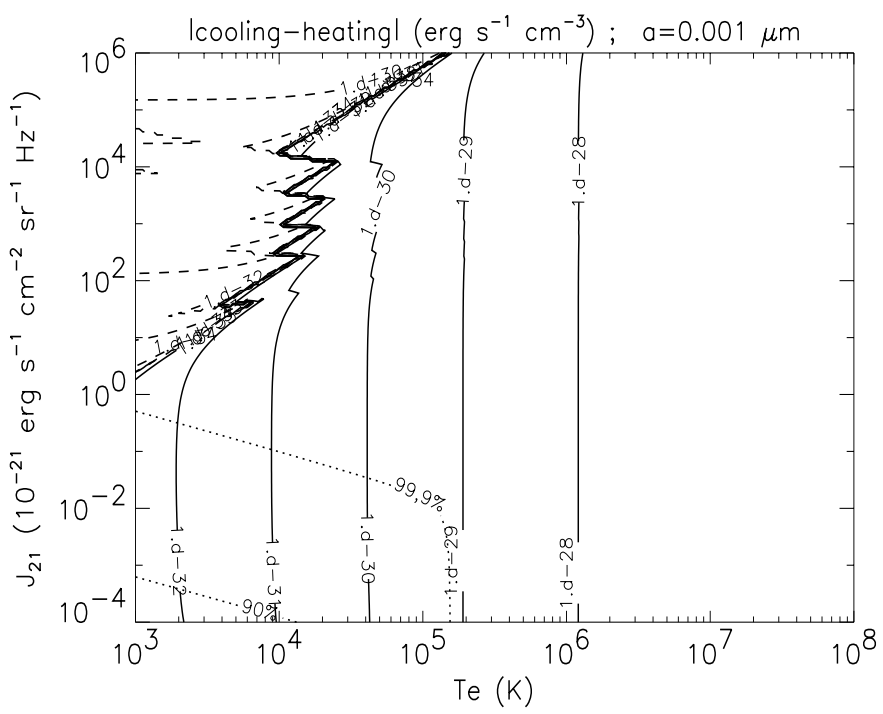

Fig. 5. The same as Fig. 2, but with a density $n_{\mathrm{H}}=10^{-3} \mathrm{~cm}^{-3}$. The level of ionisation is drawn at $99.9 \%$ and $90 \%$ (dotted line).

and the electronic collisional rate, implies only the modification of the grain charge equilibrium, but not of the photoelectric yield which is not very sensitive to the grain charge (just a factor 1 to 2 between minima and maxima of grain charge). Hence the dust cooling increases as the square of the density whereas the photoelectric dust heating is only proportional to the density. This impact is pointed out in Fig. 5, where we draw contours of the difference between the heating and cooling rate for a grain size $a=0.001 \mu \mathrm{m}$, and a hydrogen density $n_{\mathrm{H}}=10^{-3} \mathrm{~cm}^{-3}$, typical of the ICM, and $10^{3}$ times higher than in the previous cases, which are typical of the IGM. When comparing Figs. 5 and 2, we observe that the higher is the density, the larger is the domain of cooling efficiency, covering almost the whole range of UVB for the highest density. The impact of this density effect is detailed in Sect. 3.3.

\subsection{Cooling by dust and atomic processes}

As pointed out in Sect. 3.1, cooling by dust emission will be more efficient in a very hot medium, like the intracluster medium. But before we compare it with other cooling processes, we have to check that the UV background of this type of medium is weak enough to neglect the dust heating.

We have estimated the UVB in two different cases: in the first one we use the $\mathrm{cD}$ galaxy of the galaxy cluster Abell 1795 , and in the second we use the integrated field of galaxies of the Coma cluster. An estimate of the UV magnitude $m_{\mathrm{UV}}$ of the cD galaxy of Abell 1795, contained within a circle of radius 25", has been performed by Smith et al. (1997) with a far-UV filter with $\lambda=1520 \AA$ and $\Delta \lambda=356 \AA$. They have found $m_{\mathrm{UV}}=15.54 \pm 0.09 \mathrm{mag}$, corresponding to a flux $F_{\lambda}=2.21 \times 10^{-15} \mathrm{erg} \mathrm{s}^{-1} \mathrm{~cm}^{-2} \AA^{-1}$, using the relation $m_{\mathrm{UV}}=-2.5 \log \left(F_{\lambda}\right)-21.175$. We have assumed that this flux at $\lambda=1520 \AA$ is constant in the range $911 \leq \lambda \leq 2000 \AA$, and obtained an over-estimate of the UV energy density of this object in the range 6-13.6 eV, given by $u \leq 2.18 \times 10^{-14} \mathrm{erg} \mathrm{cm}^{-3}$. This energy density is obtained within the $\mathrm{cD}$ galaxy or in its close neighbourhood. This gives $G \leq 0.4$, and then $J_{21} \leq$ 18.86. For the second case, we have integrated the contribution of all the galaxies inside the field of the Coma cluster, contained within a circle of radius $50^{\prime}$, with the same method based on the photometric study performed by Donas et al. (1991) on the FOCA telescope with $\lambda \simeq 2000 \AA$ and $\Delta \lambda \simeq 150 \AA$. We have found a UV energy density $u=6.92 \times 10^{-17} \mathrm{erg} \mathrm{cm}^{-3}$, yielding $G=1.3 \times 10^{-3}$ and $J_{21}=0.061$. These two cases are consistent with a weak UV background inside the intracluster medium. Using the set of Figs. 2-5, we can easily check that the domain defined by $J_{21} \leq 100$ and $T_{\mathrm{e}} \geq 10^{6} \mathrm{~K}$ is totally included within the domain dominated by the dust cooling. This confirms our assumption of neglecting the UVB in first order in the intracluster medium.

The model used to compare the cooling by dust emission and the other atomic cooling processes is that given by Cen (1992), an updated version of the Black (1981) model. The intergalactic gas is modeled by a mixture of hydrogen and helium in cosmic proportion $(X=0.75, Y=0.25)$, with a dust-to-gas mass ratio fixed at $Z_{\mathrm{d}}=10^{-4}$. The processes taken into account are the recombination of HII, HeII, and HeIII; the collisional ionisation of HI, HeI, and HeII; and the photoionisation of HI, HeI, and HeII. First the concentrations of all the particles $\left(n_{\mathrm{HI}}, n_{\mathrm{HII}}, n_{\mathrm{HeI}}, n_{\mathrm{HeII}}, n_{\mathrm{HeIII}}\right.$, and $\left.n_{\mathrm{e}}\right)$ must be calculated for the equilibrium stage, for a given temperature and background radiation field. This can be easily done, by resolving a system of six equations and six unknowns, consisting of three conservation equations (one for each species HI, HeI, and $\mathrm{HeII}$ ), two mass conservation equations (for $\mathrm{H}$ and $\mathrm{He}$ ), and one neutrality equation. This system can be reduced to a polynomial of 4th order in $n_{\mathrm{e}}$, and solved numerically. This step determines the level of ionisation of the gas. The cooling function is then determined for a wide temperature range $10^{2} \mathrm{~K} \leq T \leq 10^{9} \mathrm{~K}$, by summing up the contributions by all the cooling processes: recombination, collisional ionisation, collisional excitation, bremsstrahlung, and dust emission as described in Eq. (10). 


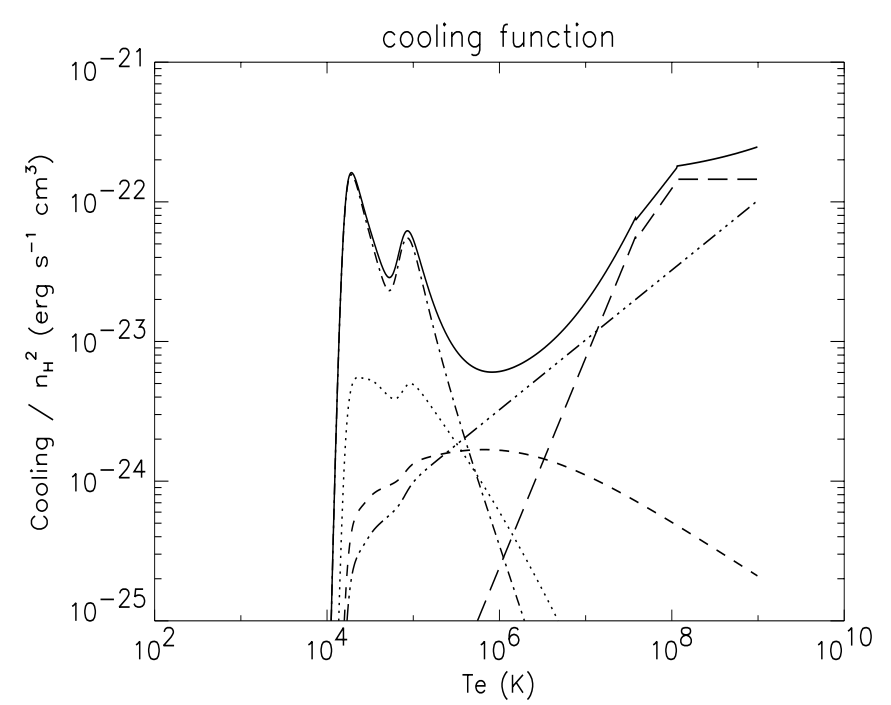

Fig. 6. Total cooling rate $\Lambda / n_{\mathrm{H}}^{2}\left(\mathrm{erg} \mathrm{s}^{-1} \mathrm{~cm}^{3}\right)$ (solid line) in the ICM, with a dust-to-gas mass ratio of $Z_{\mathrm{d}}=10^{-4}$ and a grain size $a=0.5 \mu \mathrm{m}$. Individual contributions are: collisional ionisation (dotted), recombination (dashed), collisional excitation (dash dot), bremsstrahlung (dash dot dot), dust emission (long dashes).

All these individual cooling function curves are plotted in Fig. 6, for the special case of a dust model restricted to a single grain size $(a=0.5 \mu \mathrm{m})$, and without any UV radiation field (i.e. without photoionisation) as justified above. We see that the atomic mechanisms of cooling are dominant in the range $10^{4} \mathrm{~K} \leq T \leq 10^{6} \mathrm{~K}$, where the medium is not fully ionized. For higher temperatures, $T \geq 10^{6} \mathrm{~K}$, where up to now the Bremsstrahlung was considered to be the most efficient cooling mechanism, the dust is the dominant cooling factor, as expected from the discussion in Sect. 2. Another interesting point is the dependence of the cooling function on the grain size. The dust cooling function keeps the same absolute level when the grain size decreases (from $a=0.25 \mu \mathrm{m}$ to $a=0.005 \mu \mathrm{m}$ ), but this cooling effect becomes important already at lower temperatures, filling the less efficient cooling gap between atomic and bremsstrahlung cooling (cf. Fig. 7). This means that the temperature range of efficient dust cooling is $10^{6} \mathrm{~K} \leq T \leq 10^{8} \mathrm{~K}$, depending on the grain size distribution.

\subsection{Time and spatial domains of dust cooling efficiency}

In the two previous Sects. 3.1 and 3.2, we have explored how the dust cooling behaves with respect to the temperature and density conditions. These properties can be understood as time and spatial domains.

First, the temperature range of dust cooling efficiency is $10^{6} \mathrm{~K} \leq T \leq 10^{8} \mathrm{~K}$, which is typical of the ICM. Second, we have shown that the ratio between the dust cooling and heating efficiencies becomes higher when the density increases. This again is in favor of the ICM. Thus, the ICM is the place where the dust cooling could play an important role.

Regarding time now, the previous remark on the density dependance of cooling upon heating efficiency means also that

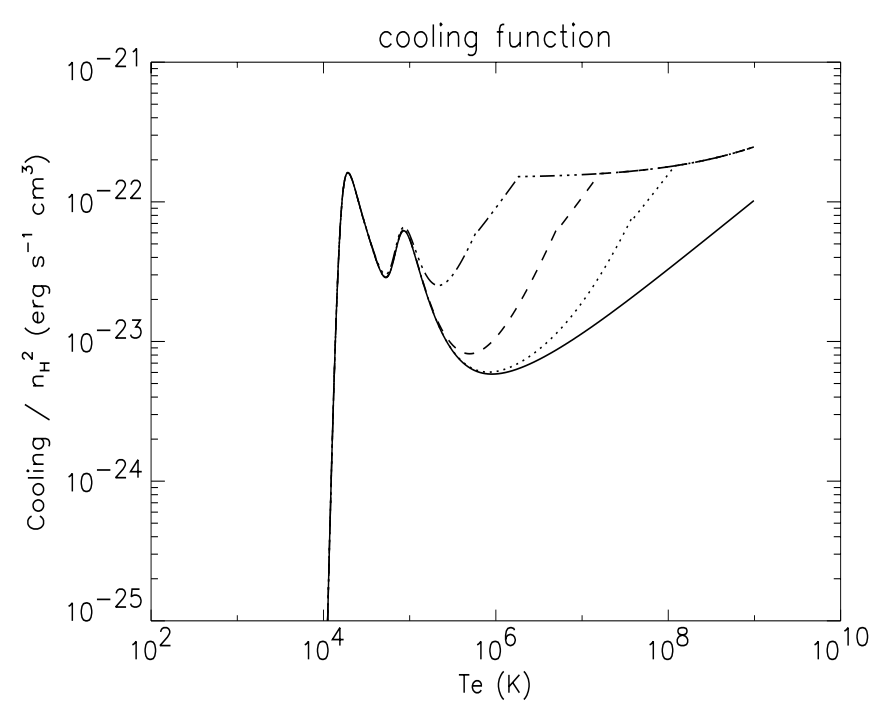

Fig. 7. Total cooling rate $\Lambda / n_{\mathrm{H}}^{2}\left(\mathrm{erg} \mathrm{s}^{-1} \mathrm{~cm}^{3}\right)$, for different cases: without dust cooling (solid), with dust cooling with grain size $a=0.5 \mu \mathrm{m}$ (dotted), $a=0.025 \mu \mathrm{m}$ (dashed), and $a=0.001 \mu \mathrm{m}$ (dash dot).

the dust cooling might have been much more efficient at high redshift, when the density was higher because of a smaller universe. Moreover at redshifts about $z \approx 3-5$, the star formation activity was at its maximum in the cosmic history, leading to an intense dust enrichment in the IGM due to violent galactic winds by supernovae (Springel \& Hernquist 2003). If we assume an average life time before dust destruction of $\tau_{\text {sput }}=10^{8}$ yr (Draine \& Salpeter 1979), this high dust amount at high redshift inside the IGM becomes localized in time, and cannot be extrapolated to lower redshifts. All these facts point to a higher dust-to-gas mass ratio in the IGM at higher redshift than now, leading to an increase of the dust cooling efficiency early in the cosmic history.

To conclude, dust has been able to play a role in the very early thermal history of the Universe, and especially in the dense and hot structures of the IGM, the clusters of galaxies.

\section{Dust abundance threshold}

The dust abundance in the IGM remains highly unknown. We will give here the dust abundance required to have the dust IR-luminosity equal to the X-ray luminosity. We will then check that this dust abundance threshold can be reached if one takes into account the mechanism usually admitted for dust enrichment, which is pollution by galaxies. We will finally go further and present another mechanism, which is the in situ dust-production by intergalactic objects, and estimate its contribution to the dust enrichment of the IGM.

\subsection{Dust threshold}

As already noticed in the expression of the IR luminosity (11), the dust cooling is proportional to the dust-to-gas mass ratio. Thus we can derive a dust abundance threshold that corresponds to the dust-to-gas mass ratio for which the dust cooling is equal to the free-free cooling. We neglect here other cooling processes such as recombination, collisional ionisation 


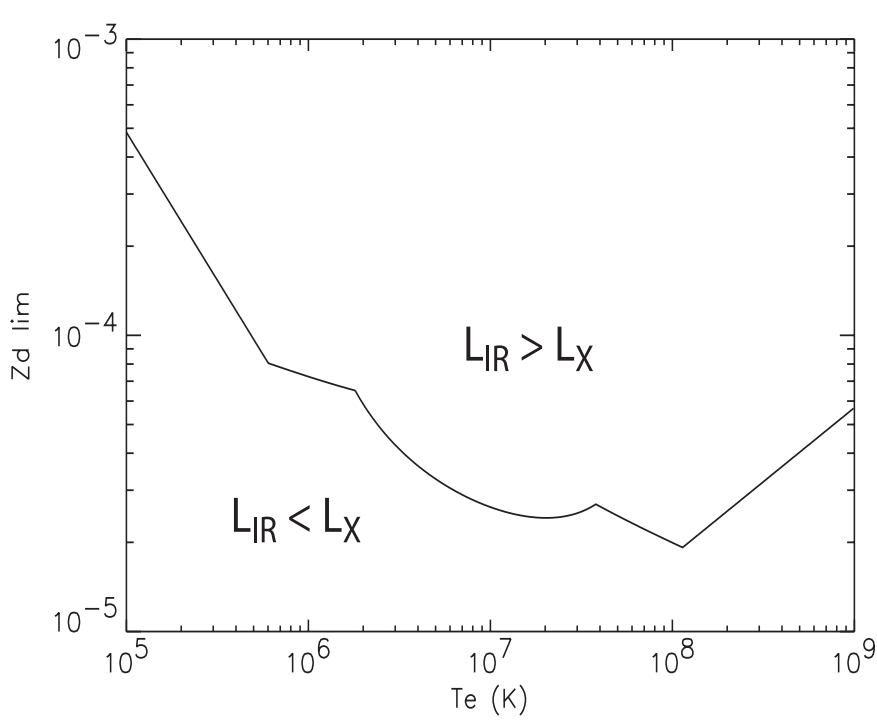

Fig. 8. Dust abundance threshold, defined as the dust-to-gas mass ratio for which the IR cooling equals the X-ray cooling

and collisional excitation in this temperature domain, in accordance with the results in Sect. 3.2. This parameter is shown in Fig. 8 for $T_{\mathrm{e}}=10^{5}$ to $10^{9} \mathrm{~K}$, and for a MRN grain model. This means that for all values of the dust-to-gas mass ratio above this curve, the IR cooling will be more efficient than the X-ray cooling. Hence considering the dust-to-gas mass ratio estimated by Stickel et al. (1998) for the Coma cluster $Z_{\mathrm{d}}=10^{-4}$, the IR cooling is more efficient than the X-ray cooling for a temperature range from $T_{\mathrm{e}}=10^{6}$ to $10^{9} \mathrm{~K}$. However, according to the simulations of Aguirre et al. (2001a) detailed in the next paragraph, the dust-to-gas mass ratio in the IGM is only about $Z_{\mathrm{d}}=2.37 \times 10^{-5}$, and the temperature range for dominance of the dust cooling extends therefore from $T_{\mathrm{e}}=10^{7}$ to $10^{8} \mathrm{~K}$. But in all cases, the cooling by dust is not a negligible process, and might even be predominant.

The key point is obviously to obtain a good estimate of the dust abundance in the IGM, and to compare it with the critical value plotted in Fig. 8. Aguirre et al. (2001a) have performed complete simulations of dust pollution of the IGM, including several ejection processes of the dust from the galaxies toward the IGM. Another dusty enrichment process is the in situ production by intergalactic stellar objects.

\subsection{Dust pollution by galaxies}

Galaxies are basically the place of birth and death of stars, and consequently of dust production. The first idea to explain the presence of dust in the intergalactic medium is the dust ejection from galaxies.

Pecker (1972) was the first to point out the dust enrichment of the IGM by radiation pressure. Aguirre et al. (2001a) have performed a complete and detailled simulation of the metal enrichment of the IGM, including the following mechanisms: dynamical removal during gravitationnal interactions (see Gnedin \& Ostriker 1997), galactic winds due to SN (see Cen \& Ostriker 1999; Aguirre et al. 2001b), and radiation pressure. The estimate of the fraction of the galactic dust expelled to the IGM during its lifetime has been given for each process: $2 \%$ to $4 \%, 2 \%$ to $25 \%$, and about $8 \%$ respectively. The dust-to-gas mass ratio of the intergalactic medium derived from this simulation is $Z_{\mathrm{d}}^{\mathrm{IGM}}=2.37 \times 10^{-5}$, which is in agreement with the presence of dust above the dust abundance threshold in the intergalactic medium restricted to the ICM, presented in Sect. 4.1 .

One can first remark that these simulations could be improved by increasing the resolution to fully understand the dynamical process of dust ejection (see Gnedin 1998). For example, simulations carried out on encounters between two galaxies reveal that about $90 \%$ of the galactic gas is removed from the parent galaxies (Duc et al. 2002).

Moreover one can stress the two following aspects implied by the mechanism of dust destruction during the transit through the galaxy: the decline of the dust-to-gas mass ratio from ISM to IGM, and the modification of the grain size distribution. The dust residing in a hot gas with $10^{6}<T<10^{9} \mathrm{~K}$ is destroyed by thermal sputtering, whose efficiency is given by the sputtering rate as quantified by Draine \& Salpeter (1979)

$\tau_{\text {sput }}=2 \times 10^{4} \mathrm{yr}\left(\frac{\mathrm{cm}^{-3}}{n_{\mathrm{H}}}\right)\left(\frac{a}{0.01 \mu \mathrm{m}}\right)$

where $\tau_{\text {sput }}$ is the lifetime until sputtering, $n_{\mathrm{H}}$ the hydrogen density, and $a$ the grain size. For typical values of the grain size from $a=0.001 \mu \mathrm{m}$ to $a=0.5 \mu \mathrm{m}(\mathrm{MRN})$, and a hydrogen density typical of dense galaxy clusters $n_{\mathrm{H}} \approx 10^{-3} \mathrm{~cm}^{-3}$, the resulting lifetime spans from $2 \times 10^{6} \mathrm{yr}$ for small grains to $10^{9} \mathrm{yr}$ for big grains. As already noted above, the small grains are relatively fragile, and can be destroyed easily. Moreover, according to a study of Ferrara et al. (1991) and Shustov \& Vibe (1995), a dust population with a size range from $0.001 \mu \mathrm{m}$ to $0.5 \mu \mathrm{m}$ in the ISM (cf. MRN), is restricted to the range $0.03 \leq a \leq 0.2 \mu \mathrm{m}$ after its transit through the galactic halo. This special destruction and selection effect could be counteracted by other dust enrichment processes like pollution by intergalactic sources as investigated in Sect. 4.3.

\subsection{Dust pollution by intergalactic sources}

The existence of a diffuse intergalactic stellar population has already been proposed by Zwicky (1951), motivated by a starlight excess between galaxies toward the Coma cluster. But the detection of isolated objects inside the intergalactic medium is quite new: intergalactic planetary nebulae in the Virgo cluster (Feldmeier et al. 1998) and in the Fornax cluster (Theuns \& Waren 1997), individual red giant stars in the Virgo cluster (Ferguson et al. 1998; Durrell et al. 2002), more recently intergalactic SNe among a sample of Abell clusters (Gal Yam et al. 2003), and HII regions in the Virgo cluster (Gerhard et al. 2002). From these observations it was concluded that as much as $20 \%$ to $50 \%$ of the stellar mass remains in the intergalactic medium. All these claims are still under discussion. The lack of observational statistics does not yet allow us to be highly confident in the estimate of the intergalactic stellar fraction. This is the reason why we decided to choose an average value of this estimate, $40 \%$, in order to derive the dust-to-gas mass ratio in 
the IGM due to this intergalactic stellar population, as detailed just below.

The stellar population outside galaxies is of a great interest for our work, because it can be considered as an additional source of dust production without any destruction processes occuring during a galactic ejection step. This in situ production could increase the dust-to-gas mass ratio of the IGM above the dust abundance threshold described above. A simple estimate of this production mechanism can be obtained using the following formula:

$\frac{M_{\text {Dust }, \text { ICSP }}}{M_{\mathrm{Gas}}}=\frac{f_{\mathrm{ICSP}} M_{\mathrm{Dust} / \mathrm{SN}} r_{S N} \tau_{\mathrm{sput}}}{M_{\mathrm{Gas}}}$

where $M_{\mathrm{D} \text {,ICSP }}$ is the dust mass produced by the IntraCluster Stellar Population, $M_{\mathrm{Gas}}$ is the gas mass of the cluster, $f_{\mathrm{ICSP}}$ is the fraction of the stellar mass present in the intracluster medium, $M_{\mathrm{D} / \mathrm{SN}}$ is the dust mass produced per SN event, $r_{\mathrm{SN}}$ is the supernova rate integrated over the galaxy cluster, and $\tau_{\text {sput }}$ is the lifetime until sputtering given by relation (16). We have chosen $f_{\mathrm{ICSP}}=40 \%$, in agreement with the proportion range given by the different authors for the observation of an intergalactic star population as discussed above. For the lifetime until sputtering an average value of $\tau_{\text {sput }}=10^{8} \mathrm{yr}$ is taken. The supernova rate $r_{\mathrm{SN}}$ is given by the integration of $\tau_{\mathrm{SN}}$, which is the number of SN per century per solar blue luminosity, over the galaxy cluster. We have chosen $\tau_{S N}=1 \mathrm{SNU}$ (SNU $=$ SN per century per $10^{10} L_{B \odot}$ ) in accordance with estimates done at low and high redshift (see Cappellaro 2001; Hardin et al. 2000; Pain et al. 1996, 2001). The blue luminosity is chosen equal to the blue luminosity of a Coma-like cluster which means $L_{B} \approx 10^{13} L_{B \odot}$, within a radius of $1 \mathrm{Mpc}$ (see White et al. 1993). With this combination we obtain a supernova rate $r_{\mathrm{SN}}=10 \mathrm{yr}^{-1}$. The gas mass contained within the same radius is then given by integration of a typical density profile (defined by King's law) with a central density $n_{0}=10^{-3} \mathrm{~cm}^{-3}$, which yield $M_{\mathrm{Gas}} \approx 2 \times 10^{13} M_{\odot}$. The last parameter of this approximation is the dust mass production per SN event. This was derived by Todini \& Ferrara (2001) for SN II and various initial metallicities and stellar masses. In our case, we have chosen an intermediate metallicity $Z=10^{-2} Z_{\odot}$, yielding an average value $M_{\mathrm{D} / \mathrm{SN}}=0.5 M_{\odot}$. Finally, using Eq. (17) and the estimates of its different parameters detailed just above, we have obtained a dust-to-gas mass ratio from the intracluster stellar population $M_{\text {Dust,ICSP }} / M_{\mathrm{Gas}} \approx 10^{-5}$, which could represent a major fraction of the total amount of dust inside the ICM. What is really of interest here is less that the amount of dust from intracluster stars is predominant compared to the galactic production, but rather that it can produce some local overdensities inside the intracluster medium, and then induce some local dust overcooling which can finally have a local impact at this scale (see Gnedin 1998).

Moreover this intergalactic production could replenish the initial grain size distribution in the range of the small grains, which are destroyed to a large fraction during the transit through the galactic medium. As pointed out by Todini \& Ferrara (2001), small grains are preferentially produced by such SNII with low metallicity, which would replenish this type of grain and balance to the destruction by sputtering.

\section{Discussion and conclusion}

\subsection{The role of dust in the evolution of the IGM}

We have shown in the previous Sects. 2 and 3 that dust can be an efficient cooling agent of the ICM when the temperature is between $T=10^{6} \mathrm{~K}$ and $T=10^{8} \mathrm{~K}$, if the dust-to-gas mass ratio exceeds $2 \times 10^{-5}$. On the other hand the dust can be a good heating agent of the IGM when the temperature is less than $T=10^{6} \mathrm{~K}$ and the UV background is strong, i.e. greater than $J_{21}=0.1$. These two opposite effects have opposite feedbacks at different scales in the Universe.

As proposed by Nath et al. (1999), the feedback of dust heating could be: at small scales, an increase of the dust abundance will heat the IGM by the photoelectric effect, increasing the temperature and thus slowing down the accretion process. This prevention of small structures accretion implies a decrease of the dust enrichment of the IGM. This type of negative feedback could have been efficient during the first ages of the universe, when the temperature was around $T \approx 10^{4}-10^{5} \mathrm{~K}$, at the beginning of small structure formation.

Following the same approach, the feedback of the dust cooling could be: at large scales, under low UV background an increase of the dust abundance implies more cooling, thus increasing the accretion rate. This acceleration of the accretion process implies an increase of star and dust formation. This positive feedback could have occured during the hierarchical clustering of the big structures, and becomes interesting when taking into account the inhomogeneous spatial distribution of the dust in the IGM pointed out in Sect. 4.3.

The inhomogeneous distribution of the dust in the IGM, implying an inhomogeneous cooling, could explain another crucial question of cosmology: the problem of preheating. The slope of the $L_{X}-T$ relation in galaxy clusters given by simple models (Kaiser 1986) is not confirmed by observations (Ponmann et al. 1996). To explain this discrepancy, it has been proposed to increase the entropy of the IGM by a "preheating phase" before cluster formation (see Valageas 2002). But the existence of such a "preheating phase" is not yet clarified, and Muanwong et al. (2001) proposed that the entropy is not increased by preheating, but by inhomogeneous radiative atomic cooling. This produces an increase by formation of sub-structures, and there is a good accordance between simulations (Muanwong et al. 2001; Pearce et al. 2000), and the fits of the slope of the $L_{\mathrm{X}}-T$ relation based on observations (Xue \& Wu 2000). Hence dust cooling could be understood in the same way, playing the same role as the atomic radiative cooling but in another temperature range, from $T=10^{6} \mathrm{~K}$ to $T=10^{8} \mathrm{~K}$, as described in Sect. 3.2.

\subsection{Perspectives}

We have presented an analytical description of dust emission in the IGM, depending on the physical properties of the dust (grain size distribution) and the medium $\left(T_{\mathrm{e}}, n_{\mathrm{e}}\right.$, $Z_{\mathrm{d}}^{\mathrm{IGM}}$ ). We have shown that the dust emission is the dominant cooling mechanism in an intermediate range of temperatures, 
$10^{6} \mathrm{~K} \leq T \leq 10^{8} \mathrm{~K}$, for a dust-to-gas mass ratio larger than $Z_{\mathrm{d}}^{\mathrm{IGM}}=2 \times 10^{-5}$.

The issue of the average value of the dust-to-gas mass ratio in the IGM is still open, and needs to be studied further, taking into account galactic and intergalactic sources. Currently such simulations are still difficult to carry out, because of the high resolution needed and the high uncertainty in the efficiency of the transport mechanisms from the galaxies into the IGM, and the unknown proportion of intergalactic sources.

The feedback of dust on the evolution of the IGM is addressed for the first time by this work. Cosmological simulations, including dust heating and cooling will be performed soon, with simple models of dust enrichment, in order to confirm the role of dust in the formation of both big and substructures of the universe.

Acknowledgements. We are endepted to the referee U. Klaas for his detailed reading which allowed us to achieve a considerable improvement for the paper. We are also thankful to Adam Walters for his contribution to the English revision of the manuscript.

\section{References}

Aguirre, A. 1999, ApJ, 525, 583

Aguirre, A., Hernquist, L., Schaye, J., et al. 2001a, ApJ, 561, 521

Aguirre, A., Hernquist, L., Katz, N., Gardner, J., \& Weinberg, D. 2001b, ApJ, 556, L11

Bajtlik, S., Duncan, R. C., \& Ostriker, J. P. 1988, ApJ, 327, 570

Bake, E. L. O., \& Tielens, A. G. G. M. 1994, ApJ, 427, 822

Bechtold, J. 1994, ApJS, 91, 1

Bennett, C. L., Halpern, M., Hinshaw, G., et al. 2003a, ApJS, 148, 1 Bennett, C. L., Hill, R. S., Hinshaw, G., et al. 2003b, ApJS, 148, 97

Black, J. H. 1981, MNRAS, 197, 553

Bogart, R. S., \& Wagoner, R. V. 1973, ApJ, 181, 609

Boyle, B. J., Fong, R., \& Shanks, T. 1988, MNRAS, 231, 897

Cappellaro, E. 2001, Mem. Soc. Astron. It., 72, 863

Cen, R. 1992, ApJS, 78, 341

Cen, R., \& Ostriker, J. P. 1999, ApJ, 519, L109

Donas, J., Milliard, B., \& Laget, M. 1991, A\&A, 252, 487

Draine, B. T., \& Lee, H. M. 1984, ApJ, 285, 89

Draine, B. T., \& Salpeter, E. E. 1979, ApJ, 231, 77

Duc, P.-A., Braine J., Liesenfeld, U., Amram, P., \& Brinks, E. 2002, Ap\&SS, 281, 347

Dupac, X., Bernard, J.-P., Boudet, N., et al. 2003, A\&A, 404, 11

Durrel, P. R., Ciardullo, R., Feldmeier, J. J., Jacoby, G. H., \& Sigurdsson, S. 2002, ApJ, 570, 119

Dwek, E., \& Werner, M. W. 1981, ApJ, 248, 138

Dwek, E. 1986, ApJ, 302, 363

Dwek, E., Petre, R., Szymkowiak, A., \& Rice, W. L. 1987, ApJ, 320, L27

Dwek, E., Rephaeli, Y., \& Mather, J. C. 1990, ApJ, 350, 104

Feldmeier, J. J., Ciardullo, R., \& Jacoby, G. H. 1998, ApJ, 503, 109

Ferguson, H. C., Tanvir, N. R., \& Von Hippel, T. 1998, Nature, 391, 461
Ferrara, A., Ferrini, F., Franco, J., \& Barsella, B. 1991, ApJ, 381, 137 Gal Yam, A., Maoz, D., Guhathakurta, P., \& Filipenko, A. V. 2003, AJ, 125, 1087

Gerhard, O., Arnaboldi, M., Freeman, K. C., \& Okamura, S. 2002, ApJ, 580, 121

Giallongo, E., Cristiani, S., D’Odorico, S., Fontana, S., \& Savaglio, S. 1996, ApJ, 466, 46

Gnedin, N. Y. 1998, MNRAS, 294, 407

Gnedin, N. Y., \& Ostriker, J. P. 1997, ApJ, 486, 581

Habing, H. J. 1968, Bull. Astron. Inst. Netherlands, 19, 421

Hardin, D., Afonso, C., Alard, C., et al., A\&A, 362, 419

Inoue, A. K., \& Kamaya, H. 2003, MNRAS, 341, 7

Kaiser, N. 1986, MNRAS, 222, 323

Karachentsev, I. D., \& Lipovetskii, V. A. 1969, SvA, 12, 909

Kitayama, T., Susa, H., Umemura, M., \& Ikeuchi, S. 2001, MNRAS, 326,1353

Mathis, J. S., Rumpl, W., \& Nordsieck, K. H. 1977, ApJ, 217, 425

Maoz, D. 1995, ApJ, 455, 115

Motta, V., Mediavilla, E., Munoz, J. A., et al. 2002, ApJ, 574719

Muanwong, O., Thomas, P. A., Kay, S. T., Pearce, F. R., \& Couchman, M. P. 2001, ApJ, 552, L27

Mushotzky, R. F., Serlemitsos, P. J., Smith, B. W., Boldt, E. A., \& Holt, S. S. 1978, ApJ, 225, 21

Nath, B. B., Sethi, S. K., \& Shchenikov, Y. 1999, MNRAS, 303, 1

Nozawa, T., Kozasa, T., Umeda, H., Maeda, K., \& Nomoto, K. 2003 , ApJ, 598, 785

Pain, R., Hook, I. M., Deustua, S., et al. 1996, ApJ, 473, 356

Pain, R., Fabbro, S., Sullivan, M., et al. 2001, AAS, 199, 1612

Pearce, F. R., Thomas, P. A., Couchman, H. M. P., \& Edge, A. C. 2000, MNRAS, 317, 1029

Pecker, J. 1972, A\&A, 18, 253

Percival, W. J., Baugh, C. M., Bland-Hawthorn, J., et al. 2001, MNRAS, 327, 1297

Pointecouteau, E. 1999, Ph.D. Thesis, Paul Sabatier University, Toulouse

Ponmann, T. J., Bourner, P. D. J., Ebeling, H., \& Bohringer, H. 1996, MNRAS, 283, 690

Shustov, B., \& Vibe, D. 1995, Astron. Rep., 39, 578

Smith, E. P., Bohlin, R. C., Bothun, G. D., et al. 1997, ApJ, 478, 516

Spergel, D., Verde, L., Peiris, H. V., et al. 2003, ApJS, 148, 175

Springel, V., \& Hernquist, L. 2003, MNRAS, 339, 312

Stickel, M., Lemke, D., Mattila, K., Haikala, L. K., \& Haas, M. 1998, A\&A, 329, 55

Stickel, M., Klaas, U., Lemke, D., \& Mattila, K. 2002, A\&A, 383, 367

Theuns, T., \& Warren, S. J. 1997, MNRAS, 284, L11

Todini, P., \& Ferrara, A. 2001, MNRAS, 325, 726

Valageas, P., Schaeffer, R., \& Silk, J. 2002, A\&A, 388, 741

Voshchinnikov, N. V., \& Khersonskij, V. K. 1984, AP\&SS, 103, 301

Weingartner, J. C., \& Draine, B. T. 2001, ApJS, 134, 263

White, S. D. M., Navarro, J. F., Evrard, A. E., \& Frenck, C. S. 1993, Nature, 366, 429

Wise, M. W., O’Connel, R. W., Bregman, J. N., \& Roberts, M. S. 1993 , ApJ, 405, 94

Xue, Y.-J., \& Wu, X.-P. 2000, ApJ, 583, 65

Zwicky, F. 1951, PASP, 63, 61

Zwicky, F. 1957, PASP, 69, 518 